\title{
Lagophthalmos surgery in leprosy: findings from a population-based survey in Korea
}

\author{
PAUL COURTRIGHT*, SUNG-HWA KIM**, \\ NARONG TUNGPAKORN ${ }^{\dagger}$, BYEONG-HEE $\mathrm{CHO}^{\dagger \dagger, \$}$, \\ YOUNG-KYU LIM ${ }^{\dagger \dagger}$, HYUN-JI LEE ${ }^{\dagger \dagger} \&$ \\ SUSAN LEWALLEN* \\ *British Columbia Centre for Epidemiologic \& International \\ Ophthalmology, University of British Columbia, Vancouver, BC, \\ Canada \\ **Catholic Skin Clinic \& Hospital, Taegu, South Korea \\ ${ }^{\dagger}$ Department of Ophthalmology, Chiang Mai University \\ ${ }^{\dagger \dagger}$ Department of Sociology, Keimyung University, Taegu, South \\ Korea \\ ${ }^{\$}$ Current address: School of Public Health, Seoul National \\ University, Seoul, South Korea
}

Accepted for publication 8 June 2001

\begin{abstract}
Summary Lagophthalmos continues to be a serious problem in cured leprosy patients. We conducted a population-based survey of lagophthalmos surgical coverage (LSC), barriers to lagophthalmos surgery and outcome of lagophthalmos surgery in leprosy patients in South Korea. In our survey, there were 60 patients with lagophthalmos who had needed surgery ( $>5 \mathrm{~mm}$ gap), 34 of whom had received surgery, resulting in a lagophthalmos surgery coverage of 57\%. Among the 34 patients who had received lagophthalmos surgery, 18 needed further surgery. Among those who had never had surgery, none of the demographic indicators predicted surgical uptake; the primary reason given for failure to have surgery was lack of knowledge about it. Outcome of surgery (by eye) showed that $29 \%$ of eyes still had a gap of $5 \mathrm{~mm}$ or more. The frequency of symptoms (tearing, blurring of vision, pain, etc.) was high. Even in settings with a good eye care infrastructure, such as Korea, uptake of surgery can still be low and results may not be satisfactory to patients. There is a need for practical guidelines for leprosy control programmes in the areas of (a) patient recognition, (b) patient education, (c) monitoring the uptake of surgery, and (d) monitoring the outcome of surgery to ensure the best possible outcome.
\end{abstract}

Correspondence to: Paul Courtright, British Columbia Centre for Epidemiologic \& International Ophthalmology, University of British Columbia, St. Paul's Hospital, 1081 Burrard Street, Vancouver, BC, V6Z 1Y6, Canada (e-mail: pcourtright@providencehealth.bc.ca) 


\section{Introduction}

We have recently demonstrated that lagophthalmos continues to be a serious problem among cured leprosy patients. ${ }^{1}$ In Korean leprosy patients (all cured mycobacteriologically for over 12 years) the presence of lagophthalmos (even on gentle closure) was associated with a 7.5fold risk of incident corneal keratitis compared to patients without lagophthalmos. In this population, although cataract was the leading cause of incident bilateral blindness $(87 \%)$ and visual impairment $(80 \%)$, corneal disease secondary to lagophthalmos was the second most frequent cause. By eye, corneal disease accounts for $19 \%$ of incident blindness (vision $<6 / 60$ ) and $56 \%$ of incident visual impairment (vision $<6 / 18$ ). Thus, corneal disease is responsible for a significant proportion of uniocular vision loss.

A survey of lagophthalmos surgical services reveals a wide variety of surgical procedures in use worldwide as well as varying indications for surgery. ${ }^{2}$ There is considerable variability in the literature regarding outcome of surgical correction of lagophthalmos; ${ }^{3-6}$ however, the findings are generally inconclusive due to small samples, inadequate follow-up, or methodological flaws. Information on surgical outcome is helpful for determining the benefits of surgical interventions as well as helping to revise surgical indicators and procedures.

There is no information in the literature documenting the lagophthalmos surgical coverage (that is, the proportion of lagophthalmos patients who have had surgery) among leprosy patients. Lagophthalmos surgical coverage should be one measure of the success of a leprosy control or blindness prevention programme. In measuring lagophthalmos surgical coverage it is important to determine why those who need surgery have not had it. These barriers can then be addressed to increase lagophthalmos surgical coverage.

In this study, we sought to determine the lagophthalmos surgical coverage, the barriers to use of lagophthalmos surgical services, and the outcome of lagophthalmos surgery.

\section{Materials and methods}

This study was undertaken in six leprosy resettlement villages in central South Korea. Leprosy is not a public health problem in Korea anymore; only 39 new leprosy cases were recognized in 1998 . There are 18,800 patients who have been treated and released, about $36 \%$ of whom live in resettlement villages. The health care infrastructure of Korea is very well developed; government and non-government programmes provide free medical (including eye) care for all leprosy patients. A well-managed tertiary eye care programme is centred at the Catholic Skin Clinic and Hospital $(\mathrm{CSCH})$ and leprosy patients from the resettlement villages under study as well as domicillary patients from surrounding countries have access to a well-trained ophthalmologist and plastic surgeon who have been providing surgery for lagophthalmos (primarily the lateral tarsal strip procedure) for the past 10 years.

In late 1988, standardized clinical examinations were undertaken on $501(83 \%)$ of 605 patients among eight leprosy resettlement villages in the area. Findings from these investigations have been reported previously. ${ }^{7-9}$ In the year following the examinations, the $\mathrm{CSCH}$ recruited an ophthalmologist to provide services for these patients. In May 1999 standardized eye examinations were repeated among the study population and the findings have been reported. ${ }^{1}$ Methods for detection of ocular pathology have been described previously. ${ }^{7-9}$ Briefly, visual acuity was taken (best eye presenting vision) using an illuminated tumbling $\mathrm{E}$ chart by a trained examiner. Clinical examinations were conducted 
by two examiners (Dr Lee Ho-Sung and SL in 1988 and NCT and SL in 1999). Corneal sensation was determined by the reaction to a cotton wisp introduced from below. Blink pattern was measured by observing the patient while he/she was unaware of being examined; it was graded abnormal when there was more than 15 seconds between blinks or if the blink was incomplete. Lagophthalmos was defined as present in 'gentle closure' when a gap was detected when a patient closed his eyes gently, as in sleep and in 'forced closure' when a gap was detected when a patient closed his eyes tightly. The gap was measured using a millimetre ruler. Presence of corneal opacity, corneal ulcer, and keratitis was also recorded. Demographic information was obtained from patient charts. Data on one patient, who had lagophthalmos secondary to a burn, was not included.

Following the clinical examinations, interviews were conducted by graduate students at the Department of Sociology, Keimyung University among all lagophthalmos patients (operated or not) to determine the barriers to surgery and satisfaction with surgery.

Lagophthalmos surgical coverage (LSC) was defined by patient rather than by eye. All patients with $5 \mathrm{~mm}$ or greater lagophthalmos in gentle or forced closure (in one or both eyes) or history of lagophthalmos surgery comprised the denominator and all patients who had had surgery for the correction of lagophthalmos (regardless of current status of lids) comprised the numerator.

We divided patients into two groups (no surgery versus surgery) and used standard univariate analyses (Students' $t$-test for continuous variables and chi-square for dichotomous variables) to evaluate the factors associated with not having surgery. Odds ratios and $95 \%$ confidence intervals were calculated. Findings from in-depth interviews on barriers to acceptance of surgery were tabulated.

Finally, outcome of surgery was evaluated, by eye rather than by patient. Clinical criteria for outcome included presence of lagophthalmos (none, 1-4 mm gap, and 5+ $\mathrm{mm}$ gap), corneal conditions (cornea ulcer or scar), and vision. Information on symptoms (excess tearing, blurring of vision, pain, disfigurement, and foreign body sensation) was also recorded at the time of examination; analysis of symptoms was by patient.

\section{Results}

There were 60 patients ( 33 men and 27 women) who either had had lagophthalmos surgery or needed surgery ( $\geq 5 \mathrm{~mm}$ gap). The 60 patients can be broken down as follows: 16 patients had had successful lagophthalmos surgery (lid gap $<5 \mathrm{~mm}$ ) and 44 patients needed surgery. Among the 44 patients needing surgery $18(40.9 \%)$ patients had a history of surgery but needed more. Thus, among the 60 patients $34(56 \cdot 7 \%, 95 \%$ confidence interval: $44 \cdot 2-69 \cdot 2 \%)$ had undergone surgery in the past; $57.6 \%$ for men and $55.6 \%$ for women. The proportion of males needing additional correction ( $15 / 19$ or $79 \%)$ outnumbered females needing additional correction $(3 / 15$ or $20 \%)$; there was no association with age or disease type. Just under half $(47.7 \%)$ of the patients needing surgery had bilateral lagophthalmos.

None of the demographic factors was associated with a failure to receive lagophthalmos surgery (Table 1). Among the 26 patients who did not have surgery, in-depth interviews were conducted with 19 patients. The reasons given by patients with lagophthalmos for not having surgery were lack of knowledge of surgery $(n=6)$, cost and distance from village $(n=4)$, service of poor quality $(n=4)$, lagophthalmos not a problem $(n=3)$ and other reasons $(n=2)$. Only nine patients $(47 \cdot 4 \%)$ knew another person who had lagophthalmos surgery. 
Table 1. Demographic and clinical factors associated with use of lagophthalmos surgical services

\begin{tabular}{|c|c|c|c|}
\hline & $\begin{array}{l}\text { Had lagophthalmos } \\
\text { surgery }(n=34)\end{array}$ & $\begin{array}{c}\text { Did not have lagophthalmos } \\
\text { surgery }(n=26)\end{array}$ & $\begin{array}{l}\text { Odds ratio (95\% } \\
\text { confidence interval) }\end{array}$ \\
\hline & No. $(\%)$ & No. & \\
\hline \multicolumn{4}{|l|}{ Age } \\
\hline$\geq 60$ years & $13(52 \cdot 0)$ & 12 & 1.0 \\
\hline$>60$ years & $20(60 \cdot 6)$ & 13 & $1.42(0.44-4.64)$ \\
\hline \multicolumn{4}{|l|}{ Gender } \\
\hline Male & $19(57.6)$ & 14 & $1 \cdot 0$ \\
\hline Female & $15(55 \cdot 6)$ & 12 & $0.92(0.29-2.91)$ \\
\hline \multicolumn{4}{|c|}{ Duration of leprosy } \\
\hline$\geq 40$ years & $9(52.9)$ & 8 & 1.0 \\
\hline $40+$ years & $24(58.5)$ & 17 & $1.25(0.35-4.53)$ \\
\hline \multicolumn{4}{|c|}{ Type of leprosy } \\
\hline MB & $22(59 \cdot 5)$ & 15 & $1 \cdot 0$ \\
\hline PB & $12(52 \cdot 2)$ & 11 & $0.74(0.23-2.41)$ \\
\hline \multicolumn{4}{|c|}{ Distance from hospital } \\
\hline Near & $22(56.4)$ & 17 & $1 \cdot 0$ \\
\hline Far & $10(55.6)$ & 8 & $0.97(0.27-3.44)$ \\
\hline
\end{tabular}

Among patients who had a history of surgery but needed more there was considerable resistance to additional surgery due to a perception that surgery did not give a good outcome.

The absence of preoperative information on these patients makes it impossible to assess whether the current findings (vision, corneal conditions) were present before surgery. Among the 34 patients with a history of surgery, in-depth interviews were conducted with 25 patients. Five patients $(20 \%)$ would not recommend surgery to others because of a failure to improve vision and continual tearing. Overall, seven patients (28\%) were not satisfied with surgery, five because their lagophthalmos recurred and two because vision worsened.

Table 2. Characteristics of eyes with a history of lagophthalmos surgery*

\begin{tabular}{lr}
\hline Clinical sign & No. $(\%)$ \\
\hline $\begin{array}{l}\text { Exposure of globe (on gentle closure) } \\
\text { No exposure }\end{array}$ & $9(21 \cdot 9)$ \\
$1-4 \mathrm{~mm}$ & $20(48 \cdot 8)$ \\
$5+\mathrm{mm}$ & $12(29 \cdot 3)$ \\
Exposure of globe (on forced closure) & $14(34 \cdot 1)$ \\
No exposure & $16(39 \cdot 0)$ \\
$1-4 \mathrm{~mm}$ & $11(26 \cdot 8)$ \\
$5+\mathrm{mm}$ & \\
Ectropion & $12(29 \cdot 3)$ \\
None & $24(58 \cdot 5)$ \\
$1 \mathrm{~mm}$ & $5(12 \cdot 9)$ \\
$2-3 \mathrm{~mm}$ & \\
Ectropion of puncta & $34(82 \cdot 9)$ \\
None & $7(17 \cdot 1)$ \\
Present & \\
\hline
\end{tabular}

* Information available on 41 patients. 
Table 3. The contribution of time elapsed since surgery on the presence of clinical characteristics in patients with a history of lagophthalmos surgery

\begin{tabular}{|c|c|c|c|}
\hline & \multicolumn{3}{|c|}{ Time elapsed since most recent surgery } \\
\hline & $\begin{array}{c}<10 \text { years } \\
\text { No. }(\%)\end{array}$ & $\begin{array}{c}10+\text { years } \\
\text { No. }\end{array}$ & OR $(95 \% \mathrm{CI})$ \\
\hline \multicolumn{4}{|c|}{ Lagophthalmos (on gentle closure) } \\
\hline$<5 \mathrm{~mm}$ & $20(68 \cdot 9)$ & 9 & $1 \cdot 11(0 \cdot 21-5 \cdot 74)$ \\
\hline$>5 \mathrm{~mm}$ gap & $8(66 \cdot 7)$ & 4 & $1 \cdot 0$ \\
\hline \multicolumn{4}{|c|}{ Lagophthalmos (on forced closure) } \\
\hline$<5 \mathrm{~mm}$ & $20(66 \cdot 7)$ & 10 & $0.75(0 \cdot 12-4.23)$ \\
\hline$>5 \mathrm{~mm}$ gap & $8(72 \cdot 7)$ & 3 & $1 \cdot 0$ \\
\hline \multicolumn{4}{|l|}{ Ectropion } \\
\hline None & $11(91 \cdot 7)$ & 1 & $7 \cdot 76(0 \cdot 81-182)^{*}$ \\
\hline Present & $17(60 \cdot 7)$ & 11 & $1 \cdot 0$ \\
\hline \multicolumn{4}{|c|}{ Ectropion of puncta } \\
\hline None & $24(75 \cdot 0)$ & 8 & $3 \cdot 0(0 \cdot 37-24 \cdot 9)$ \\
\hline Present & $3(50 \cdot 0)$ & 3 & 1.0 \\
\hline \multicolumn{4}{|c|}{ Symptoms reported } \\
\hline \multicolumn{4}{|c|}{ Excess tearing } \\
\hline Yes & $21(67.7)$ & 10 & $1 \cdot 05(0 \cdot 0-18 \cdot 1)$ \\
\hline No & $2(66 \cdot 7)$ & 1 & 1.0 \\
\hline \multicolumn{4}{|c|}{ Blurring of vision } \\
\hline Yes & $13(68 \cdot 4)$ & 6 & $1.08(0.20-5.79)$ \\
\hline No & $10(66 \cdot 7)$ & 5 & $1 \cdot 0$ \\
\hline \multicolumn{4}{|l|}{ Pain } \\
\hline Yes & $12(60 \cdot 0)$ & 8 & $0.41(0.06-2.40)$ \\
\hline No & $11(78 \cdot 6)$ & 3 & $1 \cdot 0$ \\
\hline \multicolumn{4}{|l|}{ Disfigurement } \\
\hline Yes & $8(57 \cdot 1)$ & 6 & $0.44(0.08-2.40)$ \\
\hline No & $15(75 \cdot 0)$ & 5 & 1.0 \\
\hline \multicolumn{4}{|c|}{ Foreign body sensation } \\
\hline Yes & $12(63 \cdot 2)$ & 7 & $0.62(0.11-3 \cdot 39)$ \\
\hline No & $11(73 \cdot 3)$ & 4 & $1 \cdot 0$ \\
\hline
\end{tabular}

* Fisher's exact 2-tailed $P<0.05$.

Among 48 eyes which had undergone surgery, additional information on clinical conditions was available on 41 eyes, among which $12(29.3 \%)$ had $5 \mathrm{~mm}$ or more of exposure of the globe on gentle closure (indicating the need for further surgery) (Table 2).

Among the patients who had had surgery, the frequency of symptoms reported was high; $91 \%$ reported excessive tearing, $56 \%$ reported blurring of vision, $59 \%$ reported pain, $41 \%$ reported disfigurement, and $56 \%$ reported foreign body sensation; these findings were generally not associated with duration since most recent surgery (Table 3). Ectropion was 7.7 times more common among patients who had surgery 10 or more years ago than among those who had surgery more recently.

\section{Discussion}

The prevalence of lagophthalmos in leprosy patients varies considerably among populations, primarily as a result of life expectancy, previous leprosy control efforts, and composition (leprosy type) of the population under study. None of the people in our study were new 
leprosy patients or leprosy patients currently undergoing MDT. While lagophthalmos incidence in MDT appears to be quite low there are still a considerable number of lagophthalmos patients needing surgery. ${ }^{2}$

While there are no standard criteria for defining clinically significant lagophthalmos, we chose the definition of $5 \mathrm{~mm}$ or more gap on gentle closure ${ }^{10}$ for this study. Further indications for surgery could include disfigurement and significant symptoms (e.g. excessive tearing or irritation), or a specialist's judgement that the cornea is endangered. In addition, duration of lagophthalmos needs to be considered in patient selection; patients with new onset ( $<6$ months) lagophthalmos require steroid treatment. Adoption of semi-standardized criteria for the selection of patients for surgical intervention is useful for health workers to identify patients for referral for surgery. This would also assist with monitoring lagophthalmos surgical coverage. In a recent ILEP report, it was noted that $91 \%$ of ILEP programmes report checking the lid for closure but that only $47 \%$ offer surgery. ${ }^{11}$ Clearly, the gap between reconition of lagophthalmos in the field and interventions to manage lagophthalmos is problematic.

When surgery is available, standard assessment of clinical outcome is recommended. In Korea, where the quality of surgery is quite good, $29 \%$ of eyes still had $5+\mathrm{mm}$ lid gap. Presumably the ectropion, more common among patients who had surgery over 10 years ago, is a result of ageing and gradual laxity of the lid. Excess tearing was reported by almost all patients and was responsible for most of the dissatisfaction of patients. Further investigation of the best procedures, which may vary among individual patients, is indicated. However, it is unlikely that $100 \%$ success can ever be achieved.

The fact that almost one-third of patients were not aware of the possibility of surgery for lagophthalmos is surprising; this finding suggests the need for more intense education efforts to describe the surgical procedure, benefits and risks, and the need for follow-up. In addition, the poor satisfaction reported by patients with multiple surgeries indicates the need for more intensive education of patients at the time of surgery as well as during follow-up.

The findings in Korea represent settings with aged cured leprosy patients rather than settings with active MDT programmes. Nevertheless, our findings suggest a need for creating practical guidelines for leprosy control programmes in the areas of (a) patient recognition, (b) patient education, (c) monitoring of uptake of surgery, and (d) monitoring the outcome of surgery to ensure the best possible results.

\section{Acknowledgements}

This work was supported by LEPRA, to whom we are grateful. We are also grateful for the assistance of Mrs Pak Ok-Jun and the staff at the Catholic Skin Clinic and Hospital and, most of all, to the residents of the villages.

\section{References}

${ }^{1}$ Lewallen S, Tungpakorn NC, Kim SH, Courtright P. Progression of eye disease in 'cured' leprosy patients: implications for understanding the pathophysiology of ocular disease and for addressing eyecare needs. $\mathrm{Br} \mathrm{J}$ Ophthalmol, 2000; 84: 817-821.

2 Courtright P, Lewallen S. Current concepts in the surgical management of lagophthalmos in leprosy. Lepr Rev, 1995; 66: 220-223. 
${ }^{3}$ Kim JW, Lee HS. The surgical correction of lagophthalmos using silastic band or tube. J Korean Ophth Soc, 1990; 31: 13-16.

${ }^{4}$ Weber MW, Van Soest A, Neff G et al. Results of surgical procedures for the correction of foot-drop and of lagophthalmos due to leprosy. Lepr Rev, 1992; 63: 255-262.

${ }^{5}$ Betharia SM, Bhaumik S. Evaluation of different surgical techniques in the management of paralytic ectropion in cases of leprosy. Afro-Asian J Ophthalmol, 1989; 7: 121-124.

6 Tan W. Effect of a modified temporal muscle transposition on leprous lagophthalmos. China Lepr J, 1994; 10: $147-152$

${ }^{7}$ Lewallen S, Courtright P, Lee HS. Ocular autonomic dysfunction and intraocular presence in leprosy. $\mathrm{Br} J$ Ophthalmol, 1989; 73: 946-949.

${ }^{8}$ Courtright P, Lewallen S, Lee HS. Comparison of the old and new WHO leprosy disability grading scheme for ocular disabilities. Int Ophthalmol, 1991; 15: 295-298.

9 Courtright P, Lee HS, Lewallen S. Training for primary eye care in leprosy. Bull WHO, 1990; 68: 347-351.

${ }^{10}$ Lubbers WJ, Schipper A, Hogeweg M, de Soldenhoff R. Paralysis of facial muscles in leprosy patients with lagophthalmos. Int J Lepr, 1994; 62: 220-224.

11 ILEP. ILEP Medical Commission Survey: Prevention of Disability in projects supported by ILEP Members. ILEP, 234 Blythe Road, London W14 0HJ, 1995. 